\title{
The Antrochoanal Polyp: Does it affect middle ear pressure?
}

\author{
Abhinav Srivastava ${ }^{1}$, Chander Mohan $^{2}$, Puneet shukla ${ }^{3}$ \\ ${ }^{I}$ (Assistant Professor, Department of E.N.T., Rohilkhand Medical College and Hospital, Bareilly, U.P. India) \\ 2 (Professor and Head, Department of E.N.T., Rohilkhand Medical College and Hospital, Bareilly, U.P. India) \\ ${ }_{3}^{3}$ (Post Graduate Student, Department of E.N.T., Rohilkhand Medical College and Hospital, Bareilly, U.P. India)
}

\begin{abstract}
Eustachian tube maintains middle ear pressure equal to that of atmospheric pressure and its functions is deranged due to various nasal, nasopharyngeal and palatal causes. Nasal polypi cause post nasal drip which is considered to cause Eustachian tube dysfunction. The present cross sectional study comprises of a total of 39 patients of Antrochoanal polyp with the aim to see the effect of antrochoanal polyp on middle ear pressure and to assess the status of middle ear pressure following surgery for antrochoanal polyp. Preoperative assessment of Middle Ear Pressure (MEP), revealed that, 31 subjects (79.5\%) had abnormal MEP in the ipsilateral ear, whereas 13 subjects(33.3\%) in the contralateral ear to the nasal polyp. Three weeks after surgery, MEP was found to be in the normal range in 26 subjects (66.7\%) and 31 subjects $(79.5 \%)$ in Ipsi and Contralateral ear respectively. Chronic Nasopharyngeal obstruction has a detrimental effect on middle ear pressure. Middle Ear Pressure(MEP) is deranged in patients of Antrochoanal polyp, which comes back to normal after surgery, but in patients with extension of nasal polyp to nasopharynx there are more chances of irreversible damage and persistent abnormal MEP in both ears.
\end{abstract}

Keywords: Antrochoanal Polyp, Middle Ear Pressure (MEP), Nasopharynx

\section{Introduction}

Eustachian tube maintains middle ear pressure equal to that of atmospheric pressure and allows normal respiratory secretions to pass to Nasopharynx. Its functions is deranged due to various nasal, nasopharyngeal and palatal causes. Nasal causes include Recurrent Rhinosinusitis, Deviated Nasal Septum (DNS), Nasal Polyps and other nasal masses . Nasopharyngeal causes comprise of enlarged adenoids, nasopharyngeal Angiofibroma, nasopharyngeal carcinoma. Cleft palate is one of the important cause among palatal causes. Eustachian tube function may also get deranged transiently during nasal packing and introduction of nasogastric tube and nasopharyngeal intubation. $(1,2,3)$

Chronic Rhinosinusutis is associated with inflammatory changes ranging from polypoid mucosa to gross nasal polypi. Nasal polypi cause post nasal drip which is considered to cause Eustachian tube dysfunction. The tube is frequently involved in different pathological conditions of the nasal, paranasal and nasopharyngeal cavities. Therefore, nasal obstruction can alter Eustachian tube function. Most inflammatory disorders of the middle ear are thought to be due to inadequate ventilation through the Eustachian tube(4).

The lymphatics of the middle ear and Eustachian tube course along the posterior-inferior aspect of the Eustachian tube, getting afferent from nasal cavity, paranasal sinuses, nasopharynx and adenoids. Efferent from plexus terminate in retropharyngeal lymph nodes. Inflammation and oedema in these areas cause obstruction to the flow, resulting in retrograde obstruction of tympanic and tubal lymphatics producing tubal dysfunction and middle ear effusion. Although tubal dysfunction and middle ear effusion may occur simultaneously, but effusion can occur in absence of frank obstruction of Eustachian tube lumen and development of middle ear vacuum(5).

There is no conclusive study regarding the effect of nasal polyps on the middle ear pressure. The present study has been taken up to assess the middle ear pressure in cases of Antrochoanal Polyp and to compare it with the post-op status of middle ear pressure.

\section{Methodology}

The cross- sectional study was conducted at the Department of ENT, of a tertiary care Hospital, Bareilly, Uttar Pradesh, India from April 2012 to March 2014. A total of 39 patients of Antrochoanal polyp were included in the study, to see the effect of antrochoanal polyp on middle ear pressure and to assess the status of middle ear pressure following surgery for antrochoanal polyp. The data was recorded in a predesigned performa and statistically analysed at the end of the study.

All Patients were treated with single modality of treatment i.e. FESS.

After detailed ENT Examination, routine blood and urine examination and CT Scan of Para nasal Sinuses, Pre-operative Middle ear pressure was recorded by Tympanometer- Interacoustics AT 235. The extent of the polyp i.e. whether the polyp is limited in Nasal cavity or extent to Nasopharynx was recorded. All the 
patients underwent FESS. Middle ear pressure was recorded prior to surgery, 72 hours after pack removal and at the end of third week post operative.

The patients suffering from any previous known chronic ear disease were excluded from the study.

The middle ear pressure was evaluated in terms of normal and abnormal middle ear pressure.

Abnormal Range of Middle Ear Pressure (MEP) means MEP is negative in the range of -100 to -400 dapa, whereas Normal MEP means MEP in the range of -100 to +100 dapa.

\section{Observation and Discussion}

In this study, Antrochoanal polyp was found to have slightly greater incidence in males with $53.8 \%$ of total cases in comparison with female which comprised of $46.2 \%$ cases(Table and Graph 1). Male to Female ratio was found to be $7: 6$.

Antrochoanal Polyp was more on Right side in 23 subjects(59 \%)(Table and Graph 2).

Evaluating the age group most affected, it was found that 24 cases(61.5\%) were in the age group of 2140 years, followed by 8 cases(20.5\%) in $0-20$ years age group followed by 6 cases(15.4\%) in 41-60 years and only 1 case $(2.6 \%)$ was found to be in more than 60 years of age(Table and Graph 3 ).

No gender preponderance was observed in our study with $53.8 \%$ of males and $46.2 \%$ of females, Chen et al. and orvidas et al also observed similar results in their studies $(6,7)$.

Age group most affected with Antrochoanal polyp was of 21-40 years (61.5\%) which is also observed in the study of Miguel et al(8).

Considering the extent of polyp, 14 cases (35.9\%) had nasopharyngeal extension whereas in 25 cases (64.1\%) polyp was limited to the respective nasal cavity only(Table and Graph 4).

Preoperative assessment of Middle Ear Pressure (MEP), revealed that, 31 subjects (79.5\%) had abnormal MEP in the ipsilateral ear, whereas 13 subjects(33.3\%) in the contralateral ear to the nasal polyp(Table 5 and Graph 5a). Incidently all these 13 subjects had nasopharyngeal extension of the nasal polyp, suggesting thereby that chronic nasopharyngeal obstruction has a bearing on the middle ear pressure.

On $3^{\text {rd }}$ post-op day, after Nasal Pack removal, MEP was abnormal in 33 subjects $(84.6 \%)$ in ipsilateral ear and 19 subjects (48.7) \% in Contralateral ear(Table 5 and Graph $5 b$ ).

FESS and Anterior Nasal Packing deranged the middle ear pressure further, probably due to inflammation caused due to surgical trauma and anterior nasal packing.

Three weeks after surgery, MEP was found to be in the normal range in 26 subjects $(66.7 \%)$ and 31 subjects (79.5\%) in Ipsi and Contralateral ear respectively (Table 5), meaning thereby that abnormal middle ear pressure as a result of acute and chronic nasal or nasopharyngeal obstruction is amenable to treatment and after removing the obstruction it tends to become normal.

It was also observed that in 14 cases $(35.9 \%)$ where there was nasopharyngeal extension of nasal polyp, MEP was negative in the contra-lateral ear in 13 subjects(33.3\%) (Table 4 and 5).

In 13 subjects(33.3\%), MEP was in the abnormal range even at 3 weeks post-op in the ipsilateral ear and in 8 subjects $(20.5 \%)$ in the contralateral ear. This figure was closely associated with 14 subjects (35.9\%) who had nasopharyngeal extention, indicating that those patients who had extention of polyp in the nasopharynx are more prone for irreversible Eustachian tube dysfunction.

Chronic nasal obstruction seems to have a detrimental effect on middle ear pressure, which may not return to normal even after removal of chronic obstruction. There might be some permanent change in peritubal nasopharyngeal mucosa due to chronic nasal obstruction, which needs to be proved histopathologically(9).

On detailed analysis of the distribution of abnormal Middle Ear Pressure(MEP) in pre-operative phase with polyp, it was observed that 18 cases(46.2\%) had MEP in the range of -200 to -101 dapa, it was followed by 7 cases $(17.9 \%)$ and 6 cases( $15.4 \%)$ lying in -300 to -201 and -400 to -301 dapa respectively(Table 6 ).

\section{Tables And Graphs}

Table and Graph-1 . Sex Ratio

\begin{tabular}{|l|l|}
\hline Sex & No. of Patients (\%) \\
\hline Male & $21(53.8 \%)$ \\
\hline Female & $18(46.2 \%)$ \\
\hline
\end{tabular}

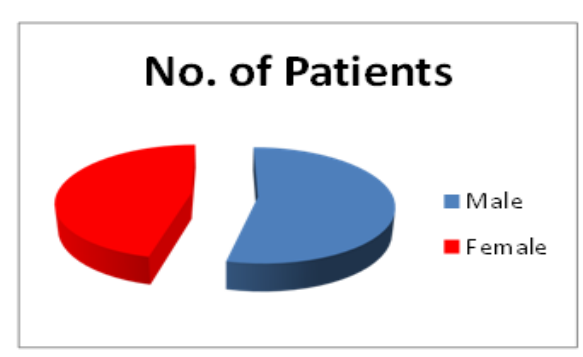


Table and Graph-2 Side predominance

\begin{tabular}{|l|l|}
\hline Side & No. of Patients $(\%)$ \\
\hline Right Side & $23(59 \%)$ \\
\hline Left Side & $16(41 \%)$ \\
\hline
\end{tabular}

Table and Graph-3. Age group distribution

\begin{tabular}{|l|l|}
\hline Age Group & No. of Patients(\%) \\
\hline $0-20$ & $8(20.5 \%)$ \\
\hline $21-40$ & $24(61.5 \%)$ \\
\hline $41-60$ & $6(15.4 \%)$ \\
\hline$>60$ & $1(2.6 \%)$ \\
\hline
\end{tabular}

Table and Graph-4. Extent of Polyp

\begin{tabular}{|l|l|}
\hline Extent of Polyp & No. of cases (\%) \\
\hline Nasal+ Nasopharynx & $14(35.9 \%)$ \\
\hline Only Nasal & $25(64.1 \%)$ \\
\hline
\end{tabular}
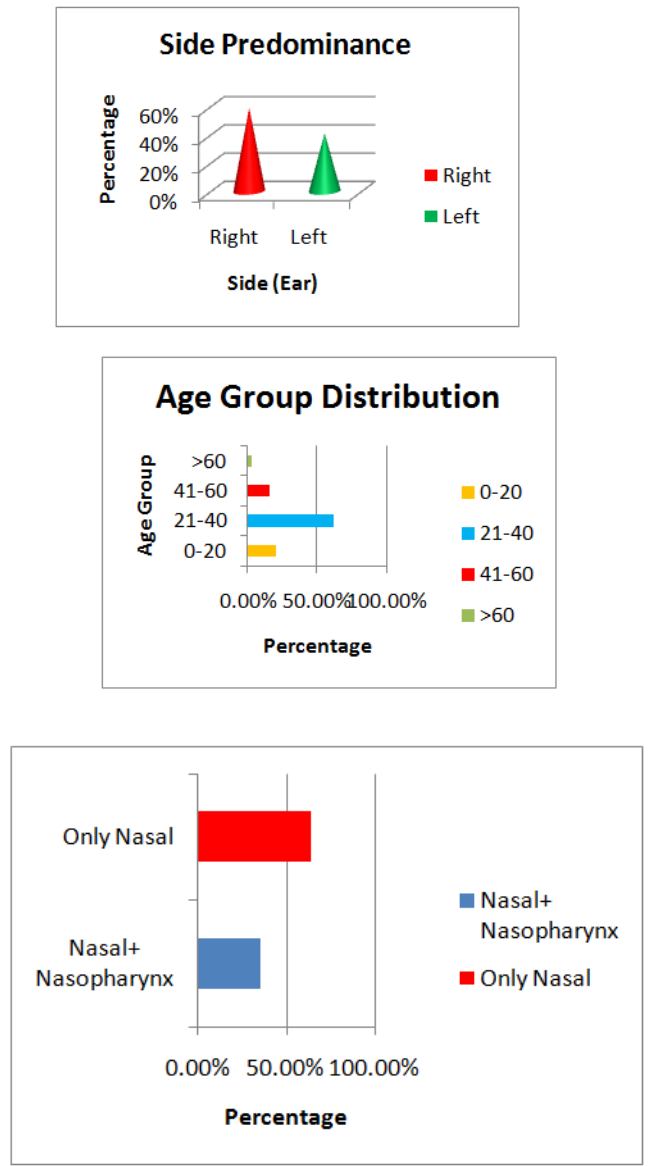

Table-5: Middle Ear Pressure

\begin{tabular}{|l|l|l|l|l|l|l|}
\hline \multirow{2}{*}{ MEP(dapa) } & \multicolumn{2}{|l|}{ No. of ears (Pre-op) } & \multicolumn{2}{l|}{ No. of ears (3 ${ }^{\text {rd }}$ day post op) } & \multicolumn{2}{l|}{ No. of ears (3 week post op) } \\
\cline { 2 - 6 } & Ipsilateral Ear & Contralateral Ear & Ipsilateral Ear & Contralateral Ear & Ipsilateral Ear & Contralateral Ear \\
\hline $\begin{array}{l}\text { Abnormal } \\
\text { Range(-100 to - } \\
400 \text { dapa) }\end{array}$ & $31(79.5 \%)$ & $13(33.3 \%)$ & $33(84.6 \%)$ & $19(48.7 \%)$ & $13(33.3 \%)$ \\
\hline $\begin{array}{l}\text { Normal Range(- } \\
100 \text { to }+100 \text { dapa }\end{array}$ & $8(20.5 \%)$ & $26(66.7 \%)$ & $6(15.4 \%)$ & $20(51.3 \%)$ & $26(66.7 \%)$ & $31(79.5 \%)$ \\
\hline
\end{tabular}

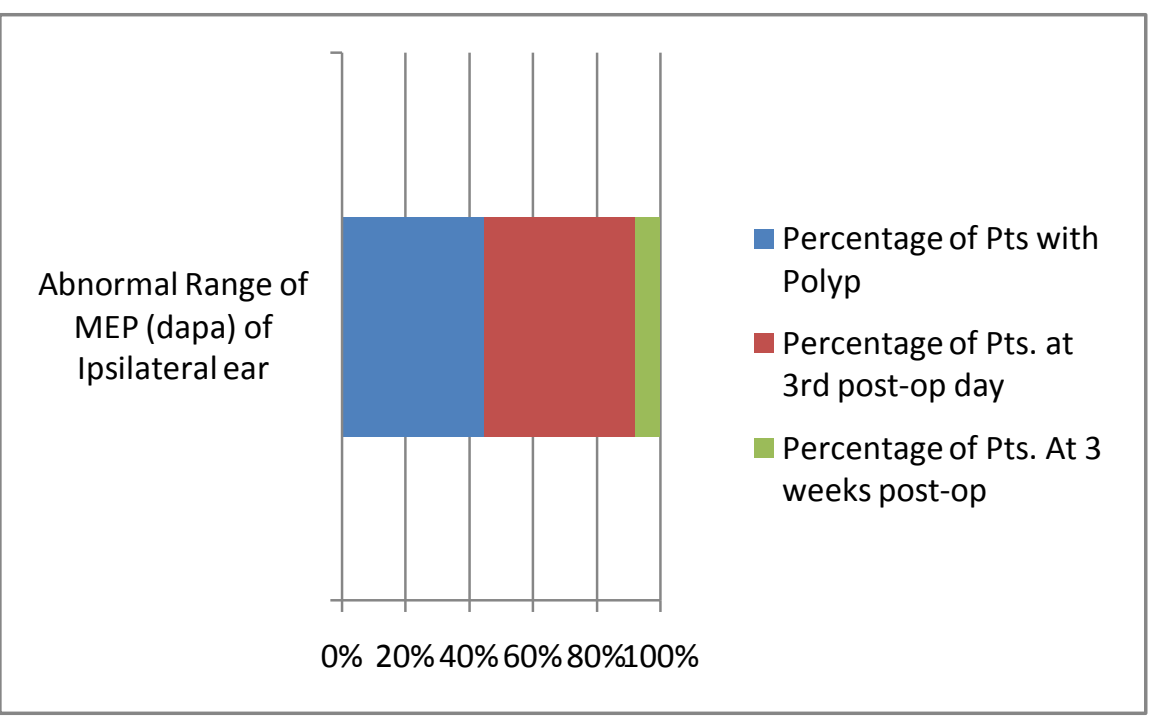

Graph 5 a: Abnormal Range of MEP(dapa) of Ipsilateral ear 


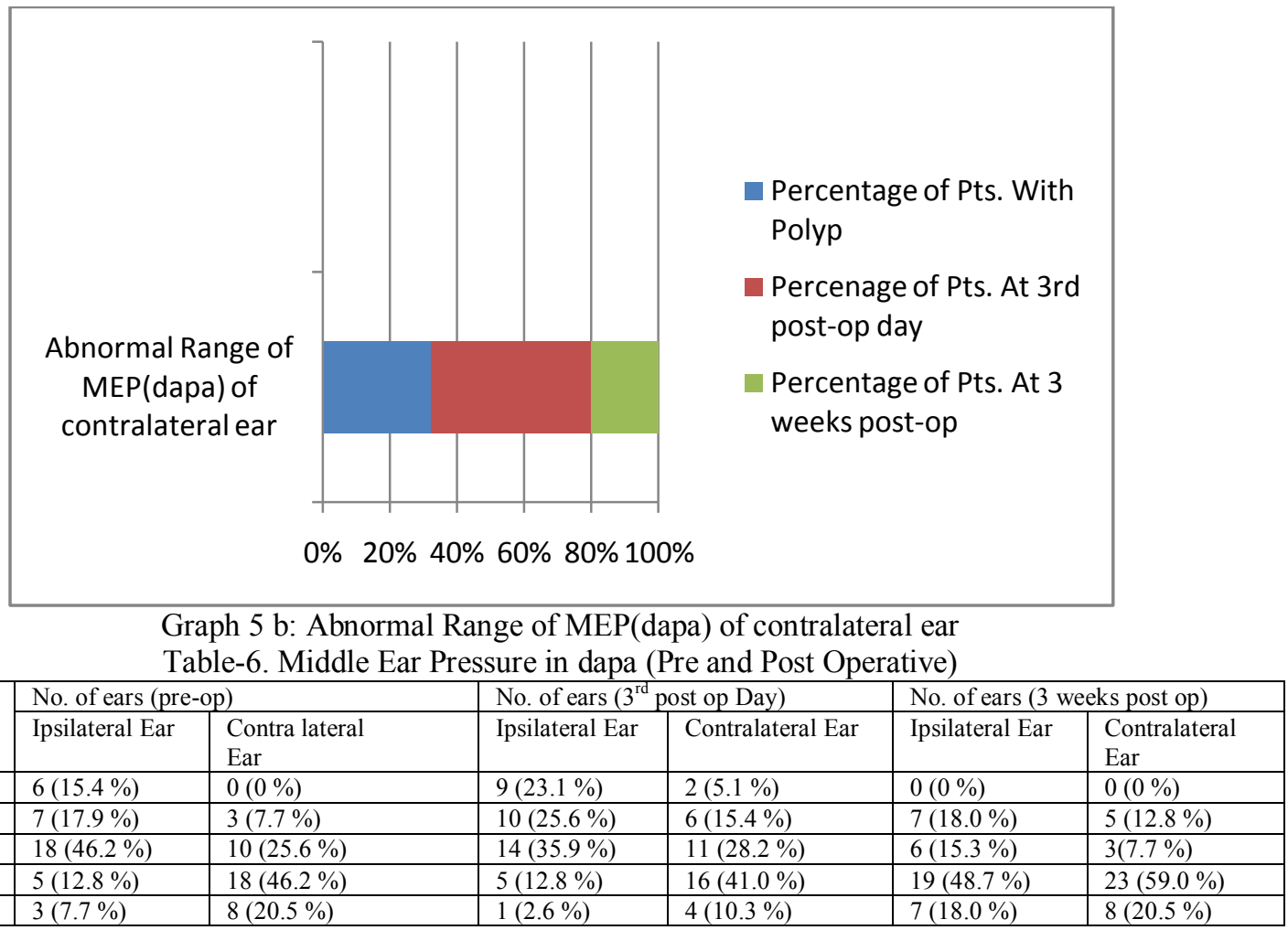

\section{Conclusion}

Chronic Nasopharyngeal obstruction has a detrimental effect on middle ear pressure. As is proved from this study that Middle Ear Pressure(MEP) is deranged in patients of Antrochoanal polyp, which comes back to normal after surgery, but in patients with extension of nasal polyp to nasopharynx there are more chances of irreversible damage and persistent abnormal MEP in both ears. It is suggested that any nasal and nasopharyngeal obstruction should be treated early to avoid irreversible changes in the Middle Ear Pressure(MEP).

\section{References}

[1]. Johannessan J, Poulsen P. The influence of anterior nasal packings on middle ear pressure. Acta Otolaryngol 1984; $97: 363-69$.

[2]. Bonding P, Tos M. Middle ear pressure during brief pathological conditions of the nose and throat. Acta Otolaryngol 1981; 92:6369.

[3]. Wake M, McCullough DE, Binnington JD. Effect of nasogastric tubes on Eustachian tube function. J Laryngol Otol 1990;104:1719.

[4]. Sadia Chaudhry, Zafar Ahmad, Faraz Basharat Khan, Mohammad Afzal. Frequency of Otitis Media in Patients of Nasal Polypi. J Ayub Med Coll Abbottabad 2010; 22(2): 83-85.

[5]. Mohan C, Saxena RK, Chauhan PG. Effect of anterior nasal packing on middle ear pressure. Indian Journal of Otolaryngology 1990; 42(3): 130-131.

[6]. Chen JM, Schloss MD, Azouz ME, Antrochoanal polyps: a 10 year retrospective study in the pediatric population with a review of literature. J Otolaryngol 1989; 18: 168-172.

[7]. Orvidas LJ, Beatty CW, Weaver AL. Antrochoanal polyps in Children. Am J Rhinol 2001; 15:321-325.

[8]. Miguel Maldonado, Asuncion Martinez, Isam Alobid, Joaquim Mullol. The Antrochoanal Polyp. Rhinology 2004;43:178-182.

[9]. Mohammad Rokanuddin Bhuiyan, Mohammad Idrish Ali, Fatema Johora, Sk. Nurul Fattah Rumi, Nazmul Huque, M. Abdullah. Effect of anterior nasal packing on middle ear pressure. Bangladesh J Otorhinolaryngol 2011; 17(2): 125-131. 